\title{
AUDITORY CENTERS IN THE ELASMOBRANCH BRAIN STEM: DEOXYGLUCOSE AUTORADIOGRAPHY AND EVOKED POTENTIAL RECORDING
}

\section{JEFFREY T. CORWIN* and R. GLENN NORTHCUTT}

Neurobiology Unit, Scripps Institution of Oceanography, Department of Neurosciences, University of California, San Diego, La Jolla, CA and Division of Biological Sciences, University of Michigan, Ann Arbor, MI (U.S.A.)

(Accepted August 6th, 1981)

Key words: audition - elasmobranchs - evolution - deoxyglucose - lateral line

\section{SUMMARY}

Elasmobranchs are sensitive to low frequency sound, and electrophysiological studies have demonstrated acoustic responses from the ear. Five primary projections from the ear to the medulla have been found, but individually they could not be identified with either the auditory or the equilibrium modality since they originate in a mixed nerve. Metabolic mapping in the brain of the thornback guitarfish with $\left[{ }^{14} \mathrm{C}\right] 2-$ deoxyglucose autoradiography and acoustic stimulation provided tentative identifications of acoustic centers in cell plate $X$ of the medial octavolateralis nucleus, the anterior octaval nucleus, the nucleus of the lateral lemniscus, and the ventromedial division of the lateral mesencephalic nucleus. Evoked potential recordings confirmed acoustic activity in those sites and additionally in the reticular formation, and the lateral granule cell mass of the auricle. In tests in the mesencephalon the evoked potential disappeared within the range of elasmobranch behavioral thresholds and when the eighth nerves were cut, but was not changed when all 4 lateral line nerves were cut. The identified acoustic centers resemble those found in auditory lemniscal pathways in mammals and other tetrapods, but the most recent ancestor common to elasmobranchs and tetrapods lived 400 million years ago. Therefore, a basic auditory lemniscal pathway may be a longstanding feature of the vertebrate brain.

* Present address: Department of Zoology, 2538 The Mall, University of Hawaii, Honolulu, HI, 96822 U.S.A. 


\section{INTRODUCTION}

Sharks are sensitive to low frequency sounds that are apparently detected as small displacements in the water around them ${ }^{1,14,19}$. At least some species are capable of detecting sound direction, and show strong behavioral responses to certain sounds resembling those produced by struggling fish 17,18 . The inner ears of those species contain two detectors that are clearly responsive to sound. the otolithic sacculus and the non-otolithic macula neglecta ${ }^{10}$. These detectors each contain thousands of hair cell receptors and provide physiologically measured sensitivity that matches behavioral sound sensitivity ${ }^{11,15}$. Auditory information from these detectors travels to the medulla, together with the equilibrium inputs from 5 vestibular detectors, in the conglomerate statoacoustic nerve.

Recently the projections of this nerve have been traced to at least 5 disting uishable sites in the elasmobranch hindbrain ${ }^{6,21}$. However, due to the conglomerate nature of the nerve and its ganglion, the actual modality of individual projections could not be determined by conventional anatomical methods.

In an attempt to identify the auditory projections of this nerve we used autoradiographic localization of $\left[{ }^{14} \mathrm{C}\right] 2$-deoxyglucose, a marker of active metabolic centers ${ }^{24,25}$. This method, together with evoked potential recording and histological examination of electrode tracks, was used to distinguish the auditory projections of the statoacoustic nerve, to map higher order auditory projections, and to begin physiological characterization of these histologically identified auditory regions.

\section{MATERIALS AND METHODS}

Eleven small thornbacks, Platyrhinoidis triseriata (Rhinobatidae, Elasmobranchii) (140-220 g) were used in autoradiographic experiments to locate acoustic centers. In order to prevent stimulation of visual pathways the eyelids were sutured closed and black neoprene occluders were glued over the closed eyes, then the animal was restrained in a net bag in a plexiglass tank for $2-3 \mathrm{~h}$. The tank contained previously aerated seawater and rested on a vibration isolated table in a commercial sound isolation chamber.

After the quiet period in the tank, the animal received a single i.v. injection of $\left[{ }^{14} \mathrm{C}\right] 2$-deoxy-D-glucose (New England Nuclear, $50 \mu \mathrm{Ci} / \mathrm{ml}$ in saline) at a dose of 20 $\mu \mathrm{Ci} / 100 \mathrm{~g}$ body weight. The animal then remained in the tank in either quiet conditions or with airborne noise bursts of $600 \mathrm{~ms}$ duration presented at a rate of $1 \mathrm{~s}^{-1}$ and at peak-to-peak sound pressures of $30-43 \mathrm{~dB}$ re $1 \mu \mathrm{bar}$ as measured in air above the head. After $60-90$ min the animal was anesthetized with tricaine methane sulfonate and decapitated. The unfixed brain was quickly removed, placed in a mold containing O.C.T. compound (Lab-Tek Products), and frozen by immersion into 2-methylbutane cooled in an ethanol-dry ice bath. The brain was then sectioned in a cryostat at $16 \mu \mathrm{m}$ thickness in the transverse plane. Every third section was collected on a prewarmed coverslip and dried on a warming plate at $50{ }^{\circ} \mathrm{C}$. Single-coated X-ray film (Kodak SB-5) was exposed to the sections for 3-9 days in a cassette and developed 
through standard procedures. The sections corresponding to certain autoradiographs were stained with cresyl violet and examined microscopically and by projection with corresponding autoradiographs.

Regional optical densities were measured in autoradiographs from comparable brain levels in two cases. In one case the animal had remained in quiet conditions and in the other the animal had been stimulated by noise bursts. For these measurements the film images were magnified approximately $10 \times$ in a photographic enlarger mounted above a densitometer. The section outlines were drawn and the densitometer output was adjusted to read $0 \%$ transmission when covered and $100 \%$ transmission when the lightest region of the brain was projected onto it. The relative percentage of transmission was then measured in distinguishable regions within an autoradiograph section and the boundaries of measured regions were outlined on the drawings. The transmission values were then converted to units of relative optical absorbance and the section drawings were charted as in Fig. 3.

Nine additional Platyrhinoidis triseriata $(320-880 \mathrm{~g})$ were used in evoked potential recording experiments. Animals were immobilized with tubocurarine chloride ( $2 \mathrm{mg} / \mathrm{kg}$ i.v.), placed in the vibration isolated tank, and ventilated with seawater flowing into one spiracle. The water level was adjusted so that the dorsal surface of the head was in air, then the brain was exposed by removing the skin and cartilage dorsal to it. Semi-microelectrodes made of stainless-steel insulated with epoxylite were advanced by hydraulic microdrive in a total of 44 tracks. Electrical activity was amplified with filtering (3-3000 $\mathrm{Hz}$ bandpass), monitored at an oscilloscope and on an audio channel, and averaged with a Nicolet 1070. After completing the penetration of a track the electrode tip position was marked at the bottom of the track or after retracting until an identifying electrical response was again recorded. Marks were placed by passing 5-10 $\mu \mathrm{A}$ of positive current through the electrode for $10-15 \mathrm{~s}$ and were developed by the Prussian blue method ${ }^{13}$. Brains were embedded in paraffin, sectioned transversely at $15 \mu \mathrm{m}$, and stained with cresyl violet. Blue electrode marks were identified in the microscope and charted by projection.

Airborne clicks produced by a large loudspeaker suspended by ropes from the ceiling above the tank were used as standard stimuli for the physiological recordings. They were repeated at $0.8 \mathrm{~s}^{-1}$ with peak-to-peak sound pressure of $45 \mathrm{~dB}$ re $1 \mu$ bar as monitored with a calibrated microphone (Bruel and Kjaer, type 4134) in air above the animal's head. In a few cases click intensity was varied in measuring thresholds. Threshold values in terms of sound pressure in air were converted to values for waterborne displacements by calibrating with a velocity hydrophone substituted in place of the animal after completion of an experiment.

\section{RESULTS}

The $\left[{ }^{14} \mathrm{C}\right] 2$-deoxyglucose method provides evidence for at least 4 specific auditory centers in the elasmobranch brain stem. Those centers show substantially sreater radiolabeling in animals exposed to sound as compared with control animals reld in quiet conditions. Fig. I illustrates experimental and control labeling in one 


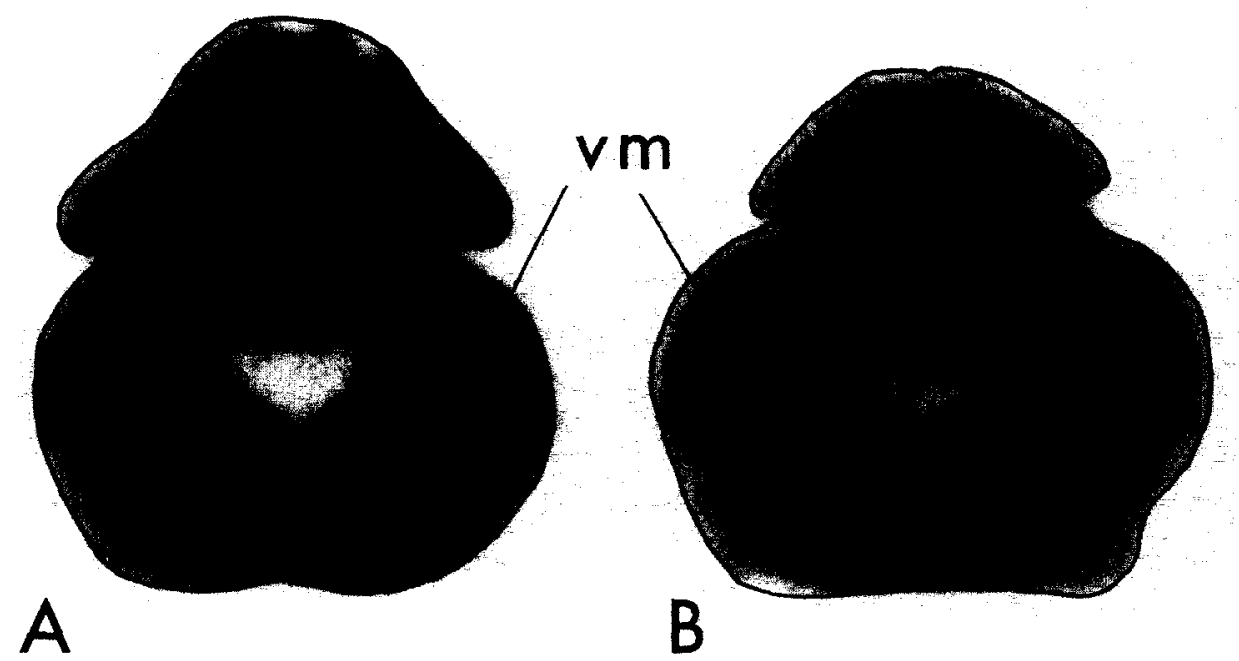

Fig. 1. X-ray film autoradiographs of transverse sections from the midbrain of a sound stimulated. thornback (A) and a control (B). VM, ventromedial division of the lateral mesencephatic nucleus.

such center, the ventromedial division of the lateral mesencephalic nucleus, a possible homologue of the torus semicircularis and inferior colliculus. As demonstrated in that figure, there is an increase in the density of labeling over this nucleus in animals exposed to sound.

The same is true for 3 additional regions labeled in autoradiographs from a sound stimulated thornback in Fig. 2. Following the nomenclature of Northcutt ${ }^{20,21}$ sections $B$ and $C$ illustrate the dense labeling of the so-called cell plate ' $X$ ' in the medial octavolateralis nuclei, section D contains the densely labeled anterior octaval nuclei and the nuclei of the lateral lemniscus, and section $E$ again shows the labeling of the lateral mesencephalic nuclei. However, in control animals held in quiet conditions each of these nuclei is still labeled more heavily than the surrounding tissue, although the labeling is not as heavy as in sound exposed animals. In our hands the 2-deoxyglucose method normally gives this type of result, a quantitative rather than a qualitative difference in the density of labeling at certain experimentally activated sites.

Densitometry of enlarged autoradiographs was conducted according to the procedure outlined in Materials and Methods in order to objectively measure the optical absorbance of these autoradiographs so as to confirm the apparent differences between control and experimental cases. The same sections printed in Fig. 2 are drawn in Fig. 3 but with regions labeled according to the measurements of relative optical absorbance. We see that cell plate $X$ of the medial octavolateralis nuclei, the anterior octaval nuclei, and the nuclei of the lateral lemniscus are in fact among the most densely labeled brain structures in the sound-exposed animal. The only other structures with comparable density of labeling are the dorsal octavolateralis nuclei, the granule cell layers in the upper leaf of the auricle, and the septal nuclei in the telencephalon, but all these appear to be non-acoustic. Recent studies indicate that these centers are involved in electroreceptive, vestibular, and olfactory pathways $s^{3,4}$ (Northcutt and Bodznick, unpublished observations). Many other structures have 
intermediate optical absorbance, including the ventromedial divisions of the lateral mesencephalic nuclei. However, in the control animal most of those regions have comparable levels of relative optical absorbance: within $25 \%$ of experimental levels. Only cell plate $X$, the anterior octaval nucleus, the lateral lemniscal nucleus, and the ventromedial division of the lateral mesencephalic nucleus show sizeable absorbance decreases in the animals held in quiet. In each of those nuclei the control absorbance is

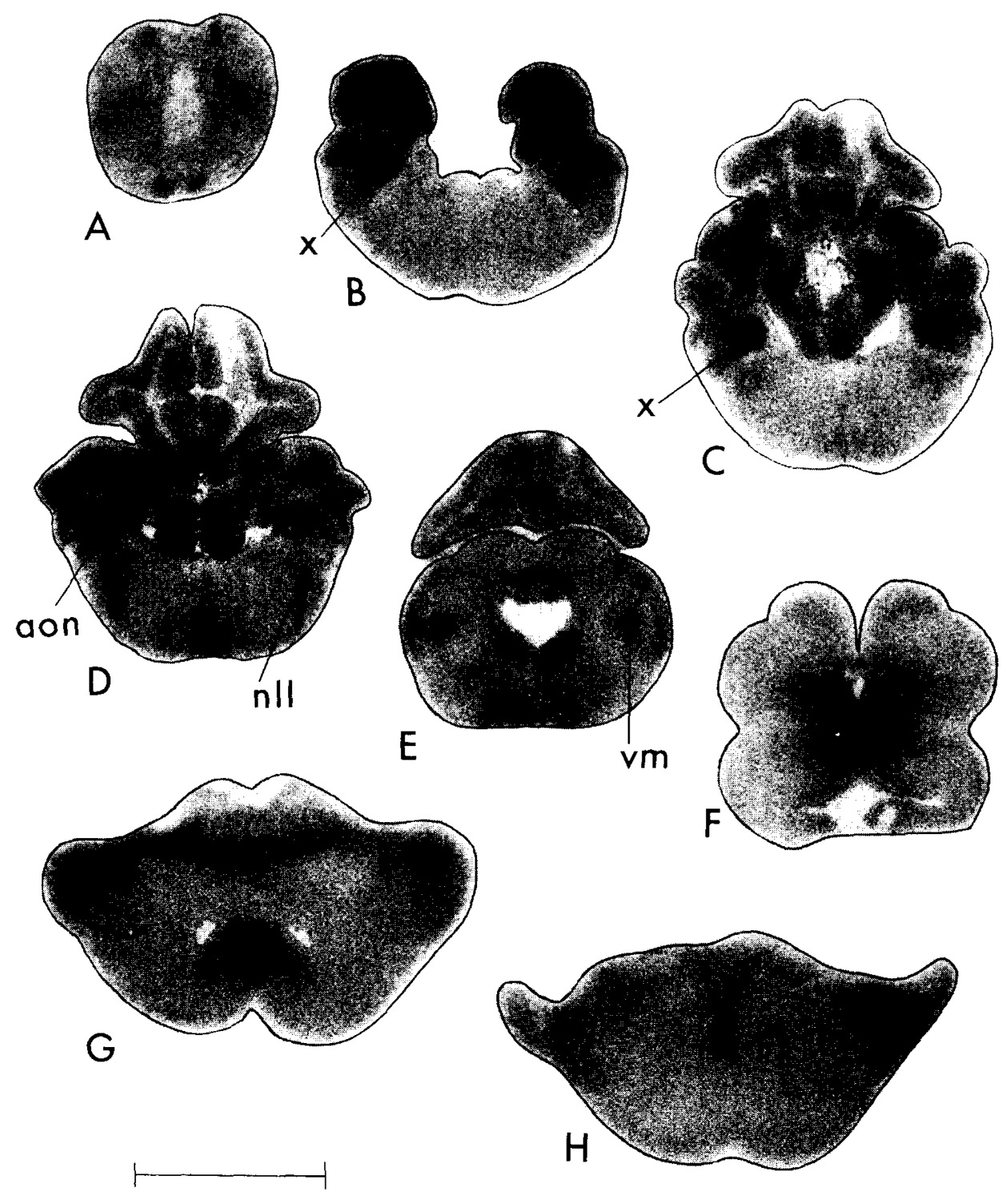

Fig. 2. X-ray film autoradiographs of transverse sections through the brain of a sound-stimulated thornback. Suspected auditory areas (areas that demonstrate marked increases in deoxyglucose activity over those in control animals) are labeled. aon, anterior octaval nucleus; nll, nucleus of the lateral lemniscus; $v m$, ventromedial division of the lateral mesencephalic nucleus; $x$, cell plate $X$ of the medial octavolateralis nucleus. Bar scale $=4 \mathrm{~mm}$. 


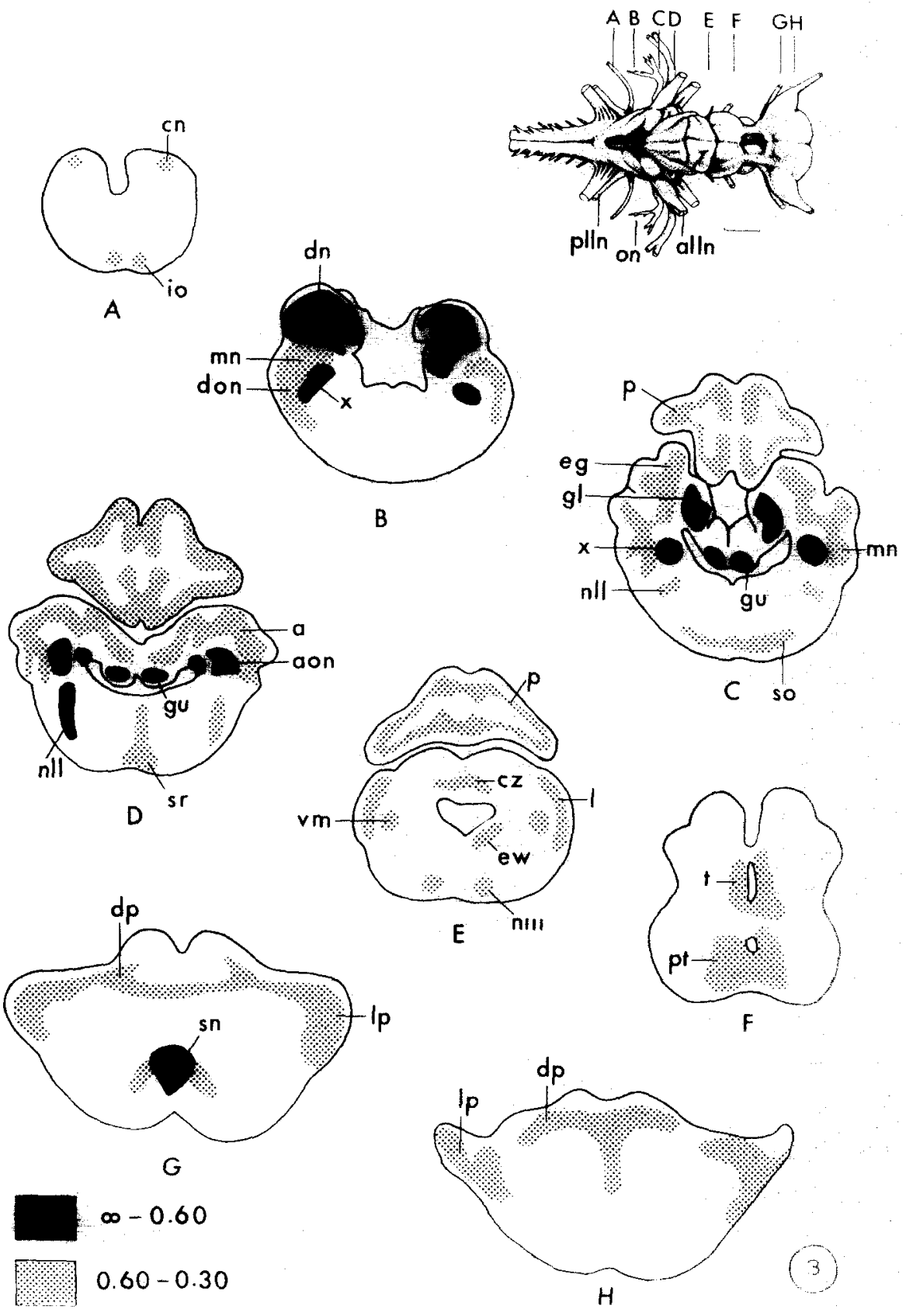

Fig. 3. Plot of deoxyglucose labeling and relative intensity (plotted as relative optical absorbance) in various brain areas of a sound stimulated thornback. a, auricle; alln, anterior lateral line nerve; aon, anterior octaval nucleus; $\mathrm{cn}$, caudal octavolateralis nucleus; $\mathrm{cz}$, central tectal zone; dn, dorsal octavolateralis nucleus; don, descending octaval nucleus; dp, dorsal pallium; eg, eminentia granularis; ew; Edinger-Westphal nucleus; gl, granule layer of lower leaf; gu, granule layer of upper leaf; io, inferior olive; 1 , lateral division of lateral mesencephalic nucleus; $1 \mathrm{p}$, lateral pallium; mn; medial octavolateralis nucleus; nll, nucleus of the lateral lemniscus; niii, oculomotor nucleus; on, octaval nerve; P, Purkinje cell layer; plln, posterior lateral line nerve; pt; posterior tuberal area; sn, septal nuclei; so, superior olive; $\mathrm{sr}$, superior raphe; $t$, thalamus; vm, ventromedial division of lateral mesencephalic nucleus; $x$, cell plate $X$ of medial octavolateralis nucleus. 
less than $50 \%$ of that measured in the sound stimulated animal. Therefore it seemed likely that these centers were receiving acoustic inputs.

Electrical recording was used in further investigations of those centers. Using a standard click stimulus, averaged evoked potentials were recorded at $500 \mu \mathrm{m}$ intervals along vertical electrode tracks throughout the brain. A total of 44 tracks were made and each acoustically active region was penetrated by at least two complete tracks. This confirmed the acoustic activity in cell plate $X$ of the medial octavolateralis nucleus, in the anterior octaval nucleus, in the nucleus of the lateral lemniscus, and in the ventromedial division of the lateral mesencephalic nucleus. It also demonstrated additional acoustically activated sites in the lateral granule cell mass and the reticular formation.

Figs. 4-7 show recordings made along representative tracks through the thornback's medulla, cerebellum, and midbrain. As illustrated in Fig. 4, acoustic evoked potentials were recorded either as slow waves alone $(<100 \mathrm{~Hz})$ or as slow waves with low amplitude spikes superimposed. Unlike the slow waves, the spike activity did not tend to be enhanced in averaged records, but spikes were clearly evident in the on-line recordings monitored on the audio channel and at the oscilloscope. Evoked spike activity was detected only in restricted regions, never extending over more than $1 \mathrm{~mm}$ in depth. Therefore particular attention was focused on spikes because their restricted detectability suggested that they were recorded only when the electrode tip was in close proximity to an active site. In contrast, slow wave activity was often recorded over $2-3 \mathrm{~mm}$ with little change in its form. In some cases the sites of evoked spike recordings coincided with the sites where slow waves reached a high point. This was always the case in mesencephalic recordings (Fig. 6), but in the medulla the tracks that passed through cell plate $X$ of the medial octavolateralis nucleus presented more complex profiles. In Figs. 4 and 5, the spike activity recorded in the vicinity of cell plate $X$ is evident even in the averaged records, but the slow wave component of the activity near cell plate $\mathrm{X}$ is no larger than that recorded more ventrally in the reticular formation. This suggests that separate generators may be giving rise to the slow wave and spike potentials recorded here. Known primary projections from the eighth nerve to the reticular formation ${ }^{6,21}$ could be involved in this acoustic response or in vestibular processing. Alternatively, the two potentials may both arise in cell plate $X$ with only the slow waves spreading to the reticular formation. The onset latencies are $8 \mathrm{~ms}$ for cell plate $X$ activity and $12 \mathrm{~ms}$ for the reticular formation slow waves.

An additional generator is also apparent in the lateral granule mass. As illustrated in Fig. 5 the electrode tracks that pass through this region record weak evoked spike activity and a small amplitude, long time-course evoked slow wave. Both forms of evoked activity disappear from the record as the electrode penetrates the crista cerebellaris, so there is little doubt that the activity originates locally in the granule cell mass.

In Fig. 6 an electrode track that passed through both the anterior octaval nucleus and the nucleus of the lateral lemniscus is illustrated. In each case we see that spike activity was recorded locally and coincident with evoked slow waves. The 

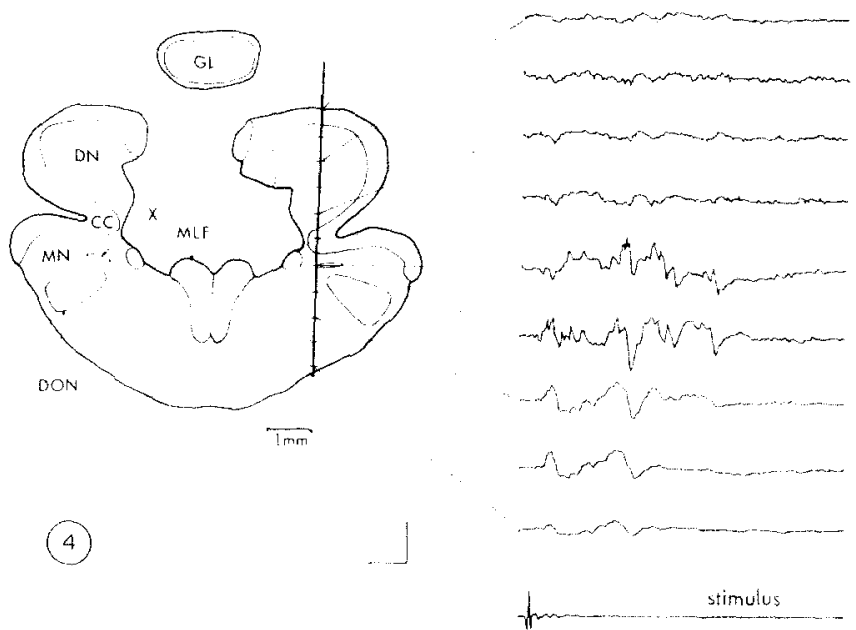

Fig. 4. A transverse section of the medulla just posterior to the statoacoustic nerve showing an electrode track and the corresponding series of evoked potentials recorded at $500 \mu \mathrm{m}$ intervals. At the level of cell plate $X$ the recording contained clear multiple unit spikes evoked by the acoustic stimuli. Ventral to that nucleus only slow waves were evoked. Each trace is an average of 32 sweeps. The lowest trace is a microphone record of the 'click' stimulus. Horizontal calibration $=25 \mathrm{~ms}$, vertical $=$ $100 \mu \mathrm{V}$. CC, cerebellar crest; DN, dorsal octavolateralis nucleus; DON, descending octaval nucleus; GL, granular layer of corpus cerebelli; MLF, medial longitudinal fasciculus; MN, medial octavolateralis nucleus; $\mathrm{X}$, cell plate $\mathrm{X}$.
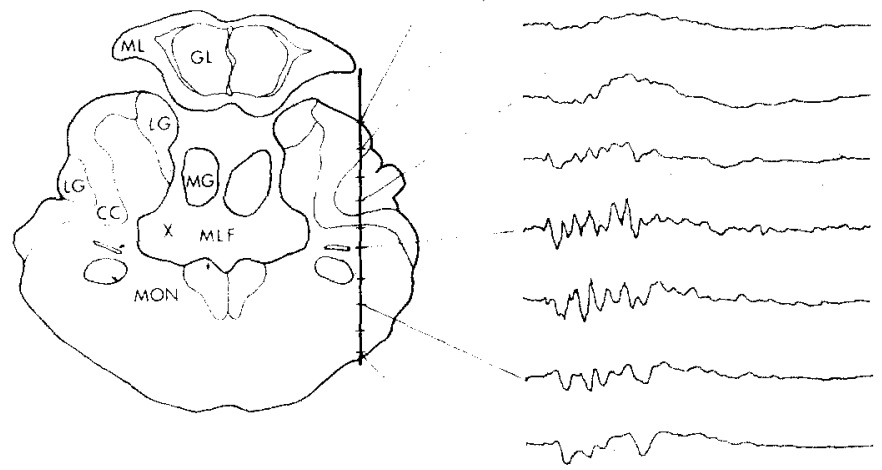

(5)

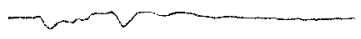

Fig. 5. A transverse section of the medulla and cerebellum at the level of the anterior portion of the entering statoacoustic nerve. In this electrode track multiple unit activity was evoked by acoustic stimuli in the lateral granule cell mass and again as the electrode passed just lateral to cell plate $\mathrm{X}$. Each trace is an average of 32 sweeps. Horizontal calibration $=25 \mathrm{~ms}$, vertical $=100 \mu \mathrm{V}$. CC, cerebellar crest; LG, lateral granule layer of lower leaf; GL, granular layer of corpus cerebelli; MG, medial granular layer of upper leaf; ML, molecular layer of corpus cerebelli; MLF, medial longitudinal fasciculus; MON, magnocellular octaval nucleus; $\mathrm{X}$, cell plate $\mathrm{X}$. 


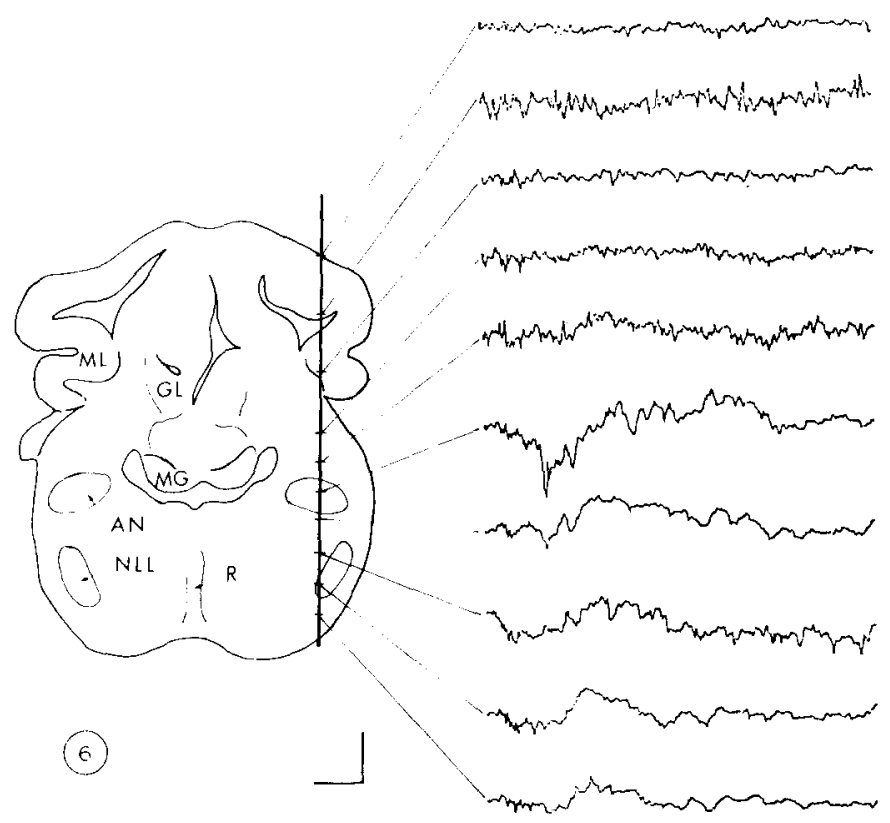

Fig. 6. A transverse section through the medulla and cerebellum anterior to the entry of the trigeminal nerve. Notice the acoustic evoked potential recorded in the anterior octaval nucleus and the low amplitude longer latency potentials recorded as this track approached the ventral portion of the nucleus of the lateral lemniscus. Each trace is the average of 32 sweeps. Horizontal calibration $=25$ $\mathrm{ms}$, vertical $=25 \mu \mathrm{V}$. AN, anterior octaval nucleus; GL, granular layer of corpus cerebelli; $\mathrm{MG}$, medial granular layer of upper leaf; ML, molecular layer of corpus cerebelli; NLL, nucleus of the lateral lemniscus; $R$, superior raphe nucleus.

activity evoked in the anterior octaval nucleus is characterized by a sudden high amplitude onset at 15-25 ms latency and by a gradual decay to near baseline. In contrast the activity evoked in the lateral lemniscal nucleus has a slow time course overall.

In Fig. 7 the activity of the ventromedial division of the lateral mesencephalic nucleus is clearly evidenced by evoked spike activity and a consistent, high amplitude slow wave. This response is characterized by a sudden positive-going onset at 13-16 ms latency followed $120 \mathrm{~ms}$ later by a negative peak of approximately equal amplitude. When the stimulus intensity was decreased the response amplitude and duration decreased and latency increased. The response disappeared when the click intensity was changed from 20 to $13 \mathrm{~dB}$ re $\mu$ bar pressure, or from approximately $4 \times 10^{-8}$ to 1 $\times 10^{-8} \mathrm{~cm}$ acoustic displacement. Behavioral hearing thresholds for other elasmobranchs range from $4 \times 10^{-8}$ to $4 \times 10^{-7} \mathrm{~cm}$ acoustic displacement ${ }^{1}$, so this evoked potential measure agrees with rough expectations.

This mesencephalic response was the most reproducible and powerful of all the responses so it was chosen for experiments designed to determine whether these acoustically evoked potentials were generated in auditory or lateral line centers. For this, recordings of spike and slow wave activity were made in the ventromedial division 


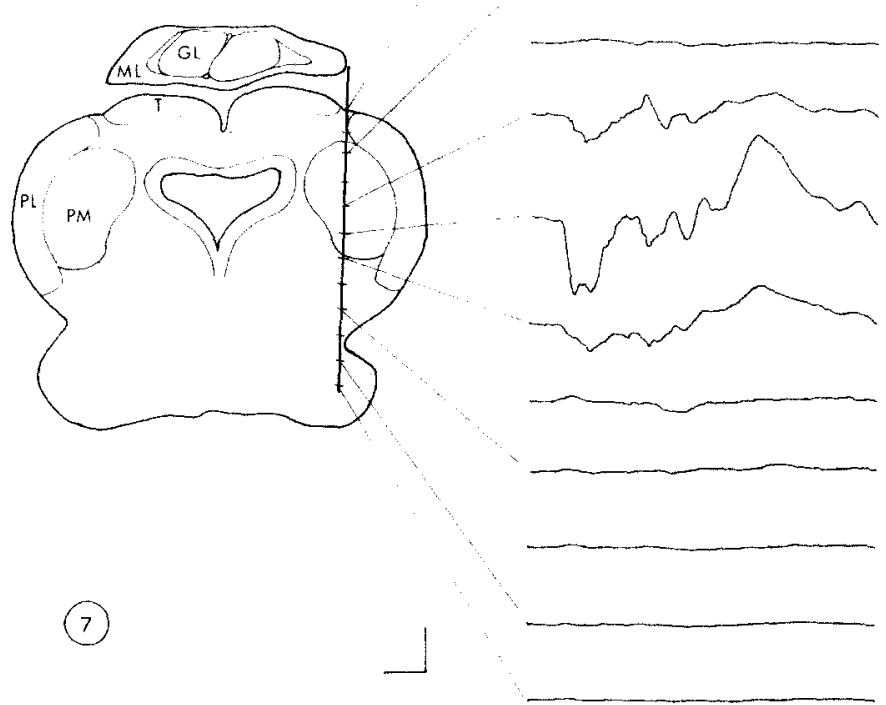

Fig. 7. A transverse section through the mesencephalon and a series of evoked potentials recorded in the track shown. Strong evoked multiple unit activity coincided in depth with the large-evoked potentials recorded in the ventromedial division of the pars medialis of the lateral mesencepablic nucleus, a suspected homologue of the torus semicircularis and inferior colliculus. Each trace is the average of 32 sweeps. Horizontal calibration $=25 \mathrm{~ms}$, vertical $=100 \mu \mathrm{V}, \mathrm{GL}$, gramular layer of corpus cerebelli; ML, molecular layer of corpus cerebelli; PL, pars lateralis of the lateral mesencephalic nucleus; PM, pars medialis (divided into dorsomedial and ventromedial divisions) of the fateral mesencephalic nucleus; $T$, superficial zone of optic tectum.

A. EIGHTH NERVES

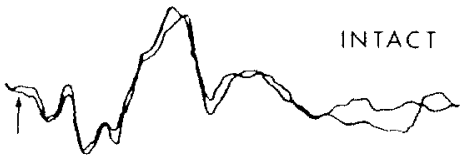

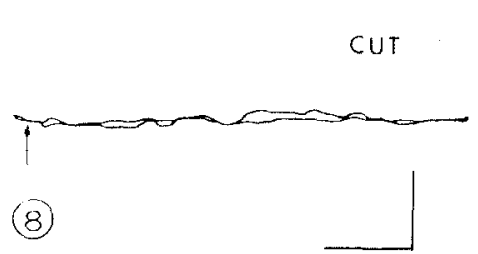

B. lateral line nerves

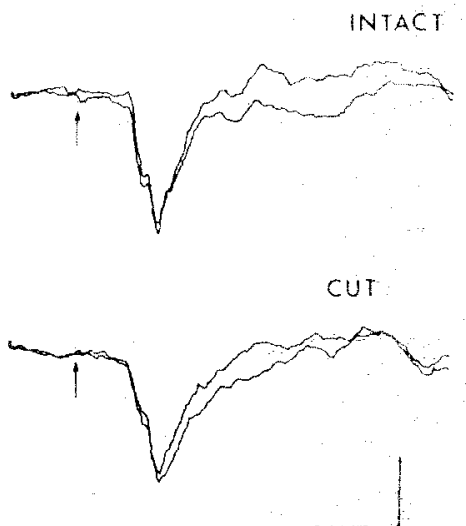

Fig. 8. Two experiments demonstrating the auditory nature of the evoked responses in the mesencephalon and their independence from the lateral line system. In A, replicate averages twere recorded before and after cutting the statoacoustic (eighth) nerves. Notice the absence of an evoked response in the lower traces. In B, replicate averages were recorded in a different animal before and after cutting the anterior and posterior lateral line nerves. Notice that the response is virtually unchanged by this manipulation. The horizontal calibration in $\mathrm{A}=100 \mathrm{~ms}$, in $\mathrm{B}=20 \mathrm{~ms}$; vertical in $\mathrm{A}=50 \mu \mathrm{V}$, in $\mathrm{B}=20 \mu \mathrm{V}$. All traces are averages of 32 sweeps with the stimulus onset marked by the arrow, 
of the lateral mesencephalic nucleus, then without moving the electrode either the statoacoustic nerves or the anterior and posterior lateral line nerves were cut bilaterally. As illustrated in Fig. 8, the response is eliminated by cutting the statoacoustic nerves, but it survives transection of all 4 lateral line nerves. We have therefore concluded that this response depends on detection of the stimulus by the ear and not by the lateral line.

\section{DISCUSSION}

The results demonstrate that acoustic stimuli can evoke increased metabolic activity and electrical activity in at least 4 nuclei in each side of the rhombencephalon and one nucleus in each side of the mesencephalon in the elasmobranch Platyrhinoidis triseriata.

Two of the activated centers, the anterior octaval nucleus and the reticular formation, receive primary projections of the eighth cranial nerve $e^{6,21}$ and both have short evoked response latencies. However, other primary projections of that nerve do not show significant increases in metabolic activity in response to these acoustic stimuli. Also, in the regions where electrode tracts penetrated these nuclei, acoustic stimuli did not evoke local activity. This negative evidence suggests that equilibrium information is processed in the magnocellular octaval nucleus, the descending octaval nucleus, and the posterior octaval nucleus. Also, throughout most portions of these nuclei, this equilibrium processing is separate from acoustic processing. However, more detailed studies are needed to positively identify the modalities of these nuclei and examine electrical activity throughout their rostrocaudal extent. Since we did not stimulate equilibrium detectors, it is not possible for us to rule out bimodal processing in cell plate $X$ of the medial octavolateralis nucleus or in the anterior octaval nucleus, but we can identify those nuclei as acoustic processing centers. It appears that equilibrium and acoustic processing systems of the thornback's brain are already at least partially separate in the medulla. This is particularly significant since the saccular and utricular detectors in the labyrinths of related elasmobranchs show combined equilibrium and acoustic sensitivity ${ }^{7,15,16}$.

These results are also relevant to the question of lateral line versus inner ear detection of sound by elasmobranchs ${ }^{22}$. Even though high intensity sound stimuli were used, there were no noticeable evoked increases in the metabolic activity measured at the two anatomically recognized projections of the mechanoreceptive lateral line system, the medial octavolateralis nucleus of the medulla and the dorsomedial division of the lateral mesencephalic nucleus. Instead, acoustic evoked activity was associated with known primary projections and suspected higher order projections in the eighth nerve processing pathway. Also, in the case of the mesencephalic center, eighth nerve destruction eliminated the electrical response, while destruction of all the lateral line nerves caused no significant change. The thresholds for acoustic evoked potentials were also low and comparable to eighth nerve and behavioral thresholds for elasmobranchs ${ }^{1,10}$. For these reasons, we believe that the acoustic centers demonstrated here are actually part of an eighth nerve mediated auditory system. Furthermore, this 
evidence and evidence from other recent studies $^{8,10}$ suggests that the lateral line system may not normally contribute to acoustic sensitivity in elasmobranchs as had been suspected ${ }^{17,18,22}$.

Finally, the anatomical positions and the sequence of evoked potential latencies in these auditory centers suggest a comparison with the auditory lemniscal pathway of amniote vertebrates. In mammals for example, that pathway is characterized by multiple centers in the medulla, a relay in the lateral lemniscal nucleus, and a sizeable projection to the inferior colliculus in the mesencephalon ${ }^{12}$. The same features appear to be present in elasmobranchs, although the number of medullary centers may be smaller here. Cell plate $\mathrm{X}$ may be a homologue of at least part of the superior olivary complex. A lateral lemniscal center lies between medullary and mesencephalic centers and the mesencephalic centers appear to be substantial in both groups. Also, a possible homology linking the lateral mesencephalic nucleus of the elasmobranchs, the torus semicircularis of other fishes, and the inferior colliculus in mammats has been proposed $^{5}$.

The similarities in this pathway as it occurs in such diverse and long separated evolutionary lines suggest that the pathway was already present in an ancestor shared by all these groups. According to most interpretations of the fossil record, the most recent ancestor common to modern elasmobranchs and the other vertebrates mentioned above lived 400 million years ago $^{23}$. It appears that a basic auditory lemniscal pathway is a longstanding feature of the vertebrate brain.

\section{ACKNOWLEDGEMENTS}

We wish to thank Dr. Theodore H. Bullock for support and encouragement. We also thank Drs. D. Bodznick, J. A. B. Gray, W. Plassman, B. L. Roberts, F. Sharp and R. W. Williamson for discussions and aid; Mary Rose Fernandez for technical assistance; and Marsha Rapson for typing. This work was supported by NIH grants to RGN, and NIH and NSF grants to Dr. Bullock. R.G.N.'s participation was made possible by a fellowship from the Guggenheim Foundation.

\section{REFERENCES}

1 Banner, A., Evidence of sensitivity to acoustic displacements in the lemon shark, Negaprion brevirostris (Poey). In P. H. Cahn (Ed.), Lateral Line Detectors, Indiana University Press, Bloomington, Indiana, 1967 , pp. 265-273.

2 Banner, A., Simple velocity hydrophones for bioacoustic application, J. Acoust. Soc. Amer., 53 (1973) 1134-1136.

3 Bodznick, D. and Northcutt, R. G., Some connections of the lateral olfactory area of the horn shark, Neurosci. Abstr., 5 (1979) 139.

4 Bodznick, D. and Northcutt, R. G., Segregation of electro- and mechanoreceptive inputs to the elasmobranch medulla, Brain Research, 195 (1980) 313-321.

5 Boord, R. L. and Northcutt, R. G., Ascending projections of anterior and posterior lateral line lobes of the clearnose skate, Raja eglanteria, Anat. Rec., 193 (1979) 487-488.

6 Boord, R. L. and Roberts, B. L., Medullary and cerebellar projections of the statoacoustic nerve of the dogfish, Scyliorhinus canicula, J. comp. Neurol., 193 (1980) 57-68.

7 Budelli, R. and Macadar, O., Statoacoustic properties of utricular afferents, $J$. Neurophysiol., 42. (1979) 1479-1493. 
8 Bullock, T. H. and Corwin, J. T., Acoustic evoked activity in the brain in sharks, J. comp. Physiol., 129 (1979) 223-234.

9 Corwin, J., Morphology of the macula neglecta in sharks of the genus Charcharhinus, J. Morph., 152 (1977) 341-362.

10 Corwin, J., Peripheral auditory physiology in the lemon shark: evidence of parallel otolithic and non-otolithic sound detection, J. comp. Physiol., (1981) 14 2A (1981) 379-390.

11 Corwin, J., Audition in elasmobranchs. In W. N. Tavolga, A. N. Popper and R. R. Fay (Eds.), Hearing and Sound Communication in Fishes, Springer-Verlag, New York, 1981, pp. 81-105.

12 Galambos, R., Neural mechanisms in audition, Laryngoscope, 68 (1958) 388-401.

13 Green, J. D., A simple microelectrode for recording from the central nervous system, Nature (Lond.), 182 (1958) 962.

14 Kelley, J. C. and Nelson, D. R., Hearing thresholds of the horn shark, Heterodontus francisci, J. Acoust. Soc. Amer., 58 (1975) 905-909.

15 Lowenstein, O. and Roberts, T. D. M., The equilibrium function of the otolithic organs of the thornback ray (Raja clavata), J. Physiol. (Lond.), 110 (1950) 392-415.

16 Lowenstein, O. and Roberts, T. D. M., The localization and analysis of the responses to vibration from the isolated elasmobranch labyrinth. A contribution to the problem of the evolution of hearing in vertebrates, J. Physiol. (Lond.), 114 (1951) 471-489.

17 Myrberg, Jr., A. A., Ha, S. J., Walewski, S. and Banbury, J. C., Effectiveness of acoustic signals in attracting epipelagic sharks to an underwater sound source, Bull. Mar. Sci., 22 (1972) 926-949.

18 Nelson, D. R., Hearing thresholds, frequency dicrimination and acoustic orientation in the lemon shark, Negaprion brevirostris (Poey), Bull. Mar. Sci., 17 (1967) 741-768.

19 Nelson, D. R. and Gruber, S. H., Sharks: attraction by low-frequency sounds, Science, 142 (1963) 975-977.

20 Northcutt, R. G., Brain organization in the cartilagenous fishes. In E. S. Hodgson and R. F. Mathewson (Eds.), Sensory Biology of Sharks, Skates, and Rays, U.S. Office of Naval Research, Arlington, Virginia, 1978, pp. 117-193.

21 Northcutt, R. G., Central auditory pathways in anamniotic vertebrates. In A. N. Popper and R. R. Fay (Eds.), Comparative Studies of Hearing in Vertebrates, Springer-Verlag, New York, 1980 , pp. $79-118$.

22 Popper, A. N. and Fay, R. R., Structure and function of the elasmobranch auditory system, Amer. Zool., 17 (1977) 443-452.

23 Romer, A. S., Vertebrate Paleontology, 3rd Edn., University of Chicago Press, Chicago, 1966.

24 Sharp, F. R., Rotation induced increases in glucose uptake of rat vestibular nuclei and vestibulocerebellum, Brain Research, 110 (1976) 141-151.

25 Sokoloff, L., Reivich, M., Kennedy, C., Des Rosiers, M. H., Patlak, C. S., Pettigrew, K. D., Sakurada, O. and Shinohara, M., The $\left[{ }^{14} \mathrm{C}\right]$ deoxyglucose method for the measurement of local cerebral glucose utilization: theory, procedure, and normal values in the conscious and anesthetized albino rat, J. Neurochem., 29 (1977) 13-26. 